\title{
Educação em saúde bucal: estudo das estratégias adotadas nas unidades de saúde do interior do Ceará
}

Oral health education: a study of the strategies adopted in the health units of the interior of Ceará

Educación en la vida bucal: las estrategias estratégicas de las unidades de educación del interior del Ceará

Téssia Richelly Nóbrega Borja de Melo ${ }^{1 *}$, Poliana de Santana Costa ${ }^{1}$, Vitor Kelven Arruda Braga ${ }^{1}$, Matheus de Almeida Germano Diniz ${ }^{1}$, Augusto Garcia de Oliveira Júnior ${ }^{1}$.

\section{RESUMO}

Objetivo: Analisar as ações de promoção e proteção em saúde bucal realizadas pelos Cirurgiões Dentistas (CD) das Equipes de Saúde da Família no interior do Ceará. Métodos: Tratou-se de um estudo quantitativo, de natureza descritiva, através da utilização de questionário, com aplicação de entrevista. A amostra foi composta por todas as Equipe de Saúde Bucal (ESB) do município $(n=16)$. Os dados foram registrados no programa SPSS (Statistical Package for Social Sciences); e descritos pelo programa Excel 2007 for Windows. Resultados: Os resultados demostraram que em 100\% das unidades eram realizadas atividades de educação em saúde, escovação supervisionada, fornecimento de escova dental e pasta fluoretada. As ações eram desenvolvidas pelos CD e Técnicos em Saúde Bucal (TSB) (58,8\%); seguidos de 29,4\%, CD e o Auxiliar de Saúde Bucal (ASB); 5,9\%, pelo TSB; e 5,9\%, por toda a ESB; sendo 58,8\%, em escolas e creches; $35,3 \%$ em escolas, creches e domicílio; e 5,9\% em unidades de saúde. Quanto aos recursos, 76,5\% utilizavam vídeos educativos; 17,6 \% álbum seriado; e 3,5 nenhum recurso. Conclusão: As ações de promoção e proteção em saúde bucal estão sendo desenvolvidas de acordo com o que preconiza a Política Nacional de Saúde Bucal, proposta pelo Ministério da Saúde.

Palavras-chave: Saúde Bucal, Promoção em Saúde, Saúde Pública.

\section{ABSTRACT}

Objective: To analyze the actions of promotion and protection in oral health performed by Dental Surgeons (CD) of the Family Health Teams in the interior of Ceará. Methods: This was a descriptive, quantitative study using a questionnaire, with interview application. The sample was carried by all the Oral Health Team (ESB) of the municipality (16). Data were recorded in the SPSS program (Statistical Package for Social Sciences); and described by the program Excel 2007 for Windows. Results: The results showed that in $100 \%$ of the units health education activities were carried out; supervised brushing, supply of toothbrush and fluoridated paste. The actions were developed by CD and Technicians in Oral Health (TSB) (58.8\%); followed by $29.4 \%$, CD and the Oral Health Assistant (ASB); $5.9 \%$, by TSB; and 5.9\%, throughout the ESB; being $58.8 \%$ in schools and day care centers; $35.3 \%$ in schools, kindergartens and domicile; and $5.9 \%$ in health units. As for resources, $76.5 \%$ used educational videos; $17.6 \%$ serial album; and 3.5 no recourse. Conclusion: The actions of promotion and protection in oral health are being developed according to what is recommended by the National Oral Health Policy, proposed by the Ministry of Health.

Key words: Oral Health, Health Promotion, Public Health.

${ }^{1}$ Faculdades Integradas de Patos (FIP, Patos, Paraíba) *E-mail: tessiaborja@yahoo.com.br

SUBMETIDO EM: 6/2019

ACEITO EM: 7/2019

PUBLICADO EM: 8/2019 


\section{RESUMEN}

Objetivo: Analizar las acciones de promoción y protección en salud bucal realizadas por los Cirujanos Dentistas (CD) de los Equipos de Salud de la Familia en el interior de Ceará. Métodos: Se trata de un estudio cuantitativo, de naturaleza descriptiva, mediante la utilización de cuestionario, con aplicación de entrevista. La muestra fue consistente por todos los Equipo de Salud Bucal (ESB) del municipio (16). Los datos se registraron en el programa SPSS (Statistical Package for Social Sciences); y descritos por el programa Excel 2007 para Windows. Resultados: Los resultados demostraron que en el 100\% de las unidades se realizaban actividades de educación en salud; cepillado supervisado, suministro de cepillo dental y pasta fluorada. Las acciones eran desarrolladas por los CD y Técnicos en Salud Bucal (TSB) $(58,8 \%)$; seguidos del 29,4\%, CD y el Auxiliar de Salud Bucal (ASB); El 5,9\%, por el TSB; y el 5,9\%, por toda la ESB; siendo $58,8 \%$, en escuelas y guarderías; $35,3 \%$ en escuelas, guarderías y domicilio; y el 5,9\% en unidades de salud. En cuanto a los recursos, el 76,5\% utilizaba vídeos educativos; $17,6 \%$ del álbum seriado; y 3,5 ningún recurso. Conclusión: Las acciones de promoción y protección en salud bucal están siendo desarrolladas de acuerdo con lo que preconiza la Política Nacional de Salud Bucal, propuesta por el Ministerio de Salud.

Palabras clave: Salud Bucal, Promoción en Salud, Salud Pública.

\section{INTRODUÇÃO}

Historicamente, a promoção de saúde foi conceituada pela primeira vez por Winslow CEA, em 1920, e Sigerist HE, em 1946. Esses autores conceituaram quatro diretrizes básicas das ciências médicas: promoção da saúde, prevenção às doenças, recuperação e reabilitação da saúde. No ano de 1965, Leavell HR e Clark EG, relataram um parâmetro para descrição da história das enfermidades, que englobou três níveis de prevenção: primário, secundário e terciário. Dentre esses níveis, a atenção primária, refere-se à manutenção da saúde, evitando-se o acometimento dos indivíduos por enfermidades (BUSS P, 2000; BUISCHI YP, 2003; SILVA RCL et al., 2006; PAULA IC e SANTOS MS, 2018).

A promoção de saúde deve acontecer em qualquer espaço da comunidade, e não somente nos postos de saúde. Associações, igrejas, praças e principalmente nas escolas, devem ser desenvolvidas atividades que visem a melhoria das condições de saúde e bem-estar da comunidade. A escola deverá receber atenção especial, visto que se pode alcançar vários grupos sociais: alunos, famílias, além de professores e funcionários, ou seja, a escola é um caminho para se abranger grande parte da comunidade (ANQUILANTE AG et al., 2003; SÁ LO e VASCONCELOS MMVB, 2009).

A inserção dos programas de saúde nas comunidades escolares tem abrangência inigualável, e pode ser ainda mais ampliada através do envolvimento dos gestores em saúde e líderes locais e comunitários. Um trabalho coletivo, com engajamento social amplo, possibilita uma maior efetividade das políticas de saúde. As atividades desenvolvidas devem ser planejadas de maneira a focar o público alvo, utilizando materiais adequados às idades escolares, e aos adultos que participarão das ações. Dessa forma haverá um maior engajamento da sociedade nas políticas de prevenção e promoção à saúde (BRASIL, 2004; BALDINI MH et al., 2003; PAULA IC e SANTOS MS, 2018).

Um dos princípios fundamentais da promoção de saúde é o desenvolvimento de ações destinadas às necessidades da população alvo. Neste sentido, o pré-requisito indispensável para o sucesso é o conhecimento da epidemiologia das doenças bucais, a realidade de cada uma das pessoas, como se organizam na comunidade e solucionam os problemas individuais e coletivos, o modo de vida, as crenças, os valores, anseios, enfim, estratégias essências para se conhecer o grupo e então promover ações em saúde adequadas à realidade (BARROS SG e CHAVES SCL, 2003; PIVEZAM G et al., 2005).

Para Buishi YP (2003), a promoção de saúde é uma ação muito mais ampla do que simplesmente repassar informações às pessoas. Orientar os pacientes de saúde bucal, sobre a forma correta de realizar a escovação dental ou a importância da utilização dos dentifrícios fluoretados durante esse processo não se traduz, 
sozinha, em promoção de saúde. Essa promoção deverá ser uma ação global que tenha como objetivo a melhoria das condições de vida e bem-estar dos indivíduos e/ ou da comunidade.

Nesse sentido, a inserção do cirurgião dentista nas equipes de saúde da família, através da implantação das equipes de saúde bucal, objetivou tornar a o serviço odontológico mais acessível aos cidadãos brasileiros, bem como quebrar o paradigma curativista estabelecido ao longo dos anos na odontologia. Para isso, as estratégias educativas, através das atividades de educação em saúde bucal, sejam nas unidades de saúde, sejam nas escolas de abrangência dessas unidades, são fundamentais para que se estabeleça uma nova saúde pautada na prevenção dos agravos à saúde bucal (BRASIL, 2004; VENÂNCIO DR et al., 2011; PAULA IC e SANTOS MS, 2018).

Frente ao exposto, o objetivo da presente pesquisa foi avaliar as ações de promoção e proteção em saúde bucal, realizadas pelos cirurgiões dentistas das equipes de saúde da família do interior do Ceará - CE.

\section{MÉTODOS}

Tratou-se de uma pesquisa do tipo quantitativa, de natureza descritiva e transversal, na qual foram avaliadas as ações de promoção e proteção em saúde bucal, realizadas nas equipes de saúde da família pelos Cirurgiões-Dentistas das Equipes de Saúde Bucal, pertencentes ao interior do estado do Ceará.

O universo foi composto de 20 unidades de saúde da família, que compunham a rede municipal de saúde, sendo a amostra 16 unidades de saúde, selecionadas por serem formadas pela equipe de atenção básica juntamente à equipe de saúde bucal (16 cirurgiões dentistas).

Foram incluídos na pesquisa todos os cirurgiões-dentistas atuantes nas equipes de saúde da família da cidade de Brejo Santo- CE, que aceitaram participar desse estudo e que assinaram o Termo de Consentimento Livre e Esclarecido. Foram excluídos os cirurgiões-dentistas atuantes em setores administrativos das equipes de saúde da família e/ou nos Centros de Especialidades Odontológicas - CEO; e aqueles que se recusaram a assinar o Termo de Consentimento Livre e Esclarecido.

Após a realização da coleta de dados, os dados foram registrados no banco de dados do programa SPSS (Statistical Package for Social Sciences) para Windows, versão 20.0. Seguidamente, as informações tabuladas foram analisadas por meio de estatística descritiva no programa Excel 2007 for Windows.

A presente pesquisa foi submetida aprovação do Comitê de Ética de Ética em Pesquisas das Faculdades Integradas de Patos no estado da Paraíba, sendo obedecidos todos os critérios prescritos pela Resolução 466/2012, do Conselho Nacional de Saúde (CNS), a qual versa sobre a ética em pesquisas com seres humanos.

Foi utilizado, como instrumento de coleta de dados, um questionário específico para esta pesquisa, baseado no trabalho de Almeida GCM e Ferreira MAF (2008), com perguntas estruturadas, em que os Cirurgiões-Dentistas foram entrevistados sobre as ações de promoção e prevenção em saúde bucal, que são realizadas em suas unidades (Figura 1). 
Figura 1 - Instrumento para Coleta de Dados (baseado no estudo de ALMEIDA GCM e FERREIRA MAF, 2008).

\section{+ैFIP}

FACULDADES INTEGRADAS DE PATOS (FIP/PB) CURSO DE BACHARELADO EM ODONTOLOGIA FORMULÁRIO PARA COLETA DE DADOS

Questionário dirigido aos Cirurgiões-Dentistas

1. O Sr (a) promove educação em saúde bucal na sua unidade de saúde?

a. ( ) $\operatorname{Sim}$ ( ) Não

2. Se o $\operatorname{Sr}(\mathrm{a})$. respondeu que SIM, responda com qual periodicidade:
a. ( ) Semanalmente
b. ( ) Quinzenalmente
c. ( ) Mensalmente
d. ( ) Bimestralmente

3. Quem participa dessa ação? (Pode marcar mais de um item)
a. ( ) Cirurgião-Dentista
b. ( ) Auxiliar de saúde Bucal
c. ( ) Técnico em Saúde Bucal
d. ( ) Professores
e. ( ) Toda a equipe de saúde bucal
f. ( ) Toda a equipe de Saúde da Família

4. Em que ambientes são realizadas essas educações em saúde bucal?
a. ( ) Escolas/creches
b. ( ) Unidades de Saúde
c. ( ) Visita Domiciliar
d. ( ) Empresas

5. Que recursos educativos o $\operatorname{Sr}(\mathrm{a})$ utiliza: (Pode marcar mais de um item)
a. ( ) Panfletos
b. ( ) Folders
c. ( ) Fantoches
d. ( ) Álbum Seriado
e. ( ) Teatro
f. ( ) Jogos Educativos
g. ( ) Vídeos
h. ( ) Nenhum

6. Existe apoio da Secretaria Municipal de Saúde para o desenvolvimento dessas atividades educativas?

$$
\text { a. ( ) } \operatorname{Sim} \text { ( )Não }
$$

7. São realizadas campanhas preventivas sobre Saúde Bucal? (Exemplo: Câncer Bucal, Tabagismo, Prevenção da Cárie, etc.)

$$
\text { a. ( ) } \operatorname{Sim} \text { ( )Não }
$$

8. Na sua unidade de saúde são realizadas ações educativo-preventivas coletivasem saúde bucal com grupos específicos, como grupos de idosos, gestantes, hipertensos, diabéticos, trabalhadores, entre outros?
a. ( ) $\operatorname{Sim}$
( ) Não

9. São feitas higiene bucal supervisionada?
a. ( ) $\operatorname{Sim}($ ) Não
b. Se o $\operatorname{Sr}($ a). respondeu que SIM, responda com qual periodicidade:
c. ( ) Semanalmente
d. ( ) Quinzenalmente
e. ( ) Mensalmente
f. ( ) Bimestralmente

10. Quem realiza essa ação?
a. ( ) Cirurgião-Dentista
b. ( ) Auxiliar de saúde Bucal
c. ( ) Técnico em Saúde Bucal
d. ( ) Professores

11. Em que ambientes são realizadas essas ações?
a. ( ) Escolas/creches
b. ( ) Unidades de Saúde
c. ( ) Visita Domiciliar
d. ( ) Empresas

12. Uma das ações de proteção à saúde segundo a Política Nacional de Saúde Bucal é a garantia à população ao acesso a escova dental e pasta dental fluoretada. No seu município é garantido esse direito?
a. ( ) $\operatorname{Sim}$
( ) Não

OBRIGADA PELA SUA PARTICIPAÇÃO!

Fonte: Dados do autor (2017). 


\section{RESULTADOS e DISCUSSÃO}

Nas tabelas que se seguem, estão descritos os resultados referentes a todas as variáveis estudas na presente pesquisa.

Tabela 1 - Distribuição das variáveis: educação em saúde; escovação supervisionada; incentivo dos gestores; atividades educativas/ preventivas em grupos específicos; acesso à escova e pasta fluoretada; campanhas preventivas.

\begin{tabular}{|c|c|c|}
\hline VARIÁVEIS & $\mathbf{N}$ & $\%$ \\
\hline Realização de Educação em Saúde & 16 & 100 \\
\hline Realização de Escovação Supervisionada & 16 & 100 \\
\hline Recebimento de Incentivo dos Gestores & 16 & 100 \\
\hline $\begin{array}{l}\text { Realização de atividades educativas/ preventivas em grupos específicos (como } \\
\text { idosos, gestantes, hipertensos, diabéticos e trabalhadores) }\end{array}$ & 16 & 100 \\
\hline $\begin{array}{l}\text { Acesso à escova dental e pasta fluoretada } \\
\text { Realizacão de campanhas preventivas (Câncer Bucal, Tabagismo, Prevenção da }\end{array}$ & 16 & 100 \\
\hline Cárie) & 15 & 97 \\
\hline
\end{tabular}

Fonte: Dados do autor (2017).

Tabela 2 - Periodicidade de realização de escovação supervisionada e atividades de educativas em Saúde Bucal.

\begin{tabular}{lcc}
\hline VARIÁVEIS/ FREQUÊNCIA & ESCOVAÇÃO SUPERVISIONADA & EDUCAÇÃO EM SAÚDE BUCAL \\
\hline Semanalmente & $11,8 \%$ & $11,8 \%$ \\
Quizenalmente & $23,5 \%$ & $23,5 \%$ \\
Mensalmente & $58,8 \%$ & $41,2 \%$ \\
Bimestralmente & $5,9 \%$ & $4,5 \%$ \\
\hline
\end{tabular}

Fonte: Dados do autor (2017).

Tabela 3 - Distribuição dos profissionais que realizam a escovação supervisionada e as atividades de educação em saúde bucal.

\begin{tabular}{lcc}
\hline $\begin{array}{l}\text { VARIÁVEIS/ } \\
\text { FREQUÉNCIA }\end{array}$ & $\begin{array}{c}\text { PROFISSIONAIS QUE REALIZAM A } \\
\text { ESCOVAÇÃO SUPERVISIONADA }\end{array}$ & $\begin{array}{l}\text { PROFISSIONAIS QUE } \\
\text { REALIZAM A EDUCAÇÃO EM } \\
\text { SAÚDE BUCAL }\end{array}$ \\
\hline CD & & $58.8,8 \%$ \\
CD e ASB & $64,7 \%$ & $29,4 \%$ \\
CD e TSB & $29,4 \%$ & $41,2 \%$ \\
TSB & $3,5 \%$ & 5,9 \\
Equipe de Saúde Bucal & Não se aplica & 5,9 \\
\hline
\end{tabular}

Fonte: Dados do autor (2017).

Tabela 4 - Distribuição das variáveis: local de realização da atividade educativa; e recurso utilizado para educação em saúde.

\begin{tabular}{|c|c|c|c|}
\hline & $\begin{array}{l}\text { Escolas e } \\
\text { Creches }\end{array}$ & $\begin{array}{l}\text { Escolas, Unidades de } \\
\text { Saúde e Domicílio }\end{array}$ & Unidades de Saúde \\
\hline $\begin{array}{l}\text { Local de realização da } \\
\text { Atividade Educativa }\end{array}$ & $58,8 \%$ & $35,3 \%$ & $5,9 \%$ \\
\hline $\begin{array}{l}\text { Recurso utilizado para } \\
\text { Educação em Saúde }\end{array}$ & $\begin{array}{l}\text { Álbum Seriado } \\
17,6 \%\end{array}$ & $\begin{array}{l}\text { Vídeo } \\
76,5 \%\end{array}$ & $\begin{array}{c}\text { Nenhum } \\
3,5 \%\end{array}$ \\
\hline
\end{tabular}

Fonte: Dados do autor (2017).

O processo ensino aprendizagem é essencial para que as políticas de educação em saúde atinjam a abrangência desejada, pois a promoção de saúde vai muito além da cura de enfermidades, almeja, principalmente, o bem-estar geral e a melhoria das condições de vida das pessoas. Programas que visam a 
comunidade escolar tendem a alcançar uma boa "fatia" da comunidade, assim como sensibilizar e influenciar no comportamento das pessoas (AQUILANTE AG et al., 2003; ALMEIDA GCM e FERREIRA MAF, 2008; PEREIRA GDS et al., 2017; PAULA IC e SANTOS MS, 2018; SILVA AH et al., 2018).

A compreensão de que a saúde bucal é parte integrante e inseparável da saúde geral, é fator indispensável para o sucesso das políticas de saúde. A infância é o período ideal para intervenções educativas, pois as crianças, em processo de formação de suas personalidades, absorverão de forma natural as ações de educação em saúde bucal, tornando os bons hábitos de higiene oral uma rotina durante seu período de crescimento (SÁ LO e VASCONCELOS MMVB, 2009; PORTO LC et al., 2015; CARVALHO IVC e CASTRO JGD, 2018).

Na primeira parte do questionário, observa-se que as equipes de Saúde Bucal estão corretamente inseridas na Estratégica de Saúde da Família. É possível também constatar o compromisso dos profissionais em participar das reuniões que são realizadas periodicamente, onde são discutidas ações de promoção e proteção em saúde, corroborando com os trabalhos de Porto LC et al., 2015; e Carvalho IVC e Castro JGD, 2018.

Vale ressaltar que os Agentes Comunitários de Saúde se desenham como um dos principais profissionais atuantes, uma vez que, conhecem profundamente a comunidade onde atuam, em razão das visitas. Entretanto, não participam de forma expressiva dessas atividades de educação e promoção em saúde bucal, assim como relataram Carvalho IVC e Castro JGD (2018), que enfatizaram que os ASBs são os profissionais que mais participam dessas atividades.

A maioria dos entrevistados, realizam atividades educativas com periodicidade regular (mensalmente, quinzenalmente, semanalmente ou bimestralmente); e sempre apenas com os integrantes da equipe de saúde bucal: a maioria com o cirurgião dentista e técnico em saúde bucal. Observa-se, portanto, que os ACSs e demais profissionais das Estratégias de Saúde da Família, não se integram a essas atividades, corroborando com os achados de Carvalho IVC e Castro JGD (2018). Esse fato pode ser avaliado como fator negativo, visto que todos os membros da equipe de saúde devem estar integrados para o desenvolvimento das ações preventivas, sejam elas em saúde bucal ou em saúde geral.

Entretanto, em relação à equipe de saúde bucal, pode-se constatar que todos os profissionais participam e/ou desenvolvem atividades de educação em saúde. Esse dado configura um avanço considerável para a odontologia no SUS, visto que denota a mudança no perfil dos profissionais, através da adoção de medidas educativas e/ou preventivas, sobrepondo-se ao método intervencionista e curativista, corroborando com os estudos de Anquilante AG et al. (2003), Barros SG e Chaves SCL (2003), e Venâncio DR et al. (2011); Pereira GDS et al., 2017; Carvalho IVC e Castro JGD (2018); Paula IC e Santos MS (2018); Silva AH et al. (2018).

Para que as ações educativas surtam efeito, faz-se necessário motivar os participantes e despertar neles o interesse em adquirir ou renovar seus conhecimentos (PORTO LC et al., 2015).

Para isso, uma das estratégias utilizadas pelos profissionais, são os recursos tecnológicos, como vídeos, álbuns seriados e materiais lúdicos, corroborando com Paula IC e Santos MS (2018). Assim como no estudo de Venâncio DR et al. (2011), o presente trabalho investigou a utilização desses recursos lúdicos nas atividades de educação em saúde bucal; tendo os profissionais relatado o uso de vídeos $(76,5 \%)$ e álbuns seriados (17,6\%).

No Brasil, as escolas aparecem como principal local social para troca e aquisição de conhecimentos, não havendo, portanto, lugar mais adequado às ações promovidas em educação preventiva (CARVALHO IVC e CASTRO JGD, 2018; SILVA AH et al., 2018).

O público presente nestas instituições não deve limitar-se somente aos estudantes, mas também a toda a comunidade do entorno escolar, pois é de fácil acesso a pais, alunos, professores, enfim, toda a comunidade escolar. Os conhecimentos adquiridos pelos participantes das atividades educativas e preventivas são disseminados por seus detentores e alcançam proporções que por si só recompensam a escolha destes locais (ANQUILANTE AG et al., 2003; BRASIL, 2004; BARROS SG e CHAVES SCL, 2003). 
Quanto ao incentivo dos órgãos governamentais, todos os profissionais afirmaram contar com o apoio da Secretaria Municipal de Saúde. Percebe-se, portanto, que há um comprometimento tanto do profissional, quanto dos órgãos governamentais em manter a população informada, investindo na educação em saúde e, consequentemente, priorizando a prevenção de agravos à saúde bucal (BARROS SG e CHAVES SCL, 2003).

Dentre as ações desenvolvidas pelas equipes de saúde bucal, destacaram-se as campanhas preventivas abordando as temáticas de Câncer Bucal, Tabagismo e Prevenção da Cárie. Além disso, a escovação supervisionada foi citada como uma das principais ações, realizada por todos os profissionais entrevistados, e de forma periódica. O acesso à escova dental foi relatado por todos os entrevistados. Observou-se, portanto, que se repete, em sua maioria, a periodicidade mensal na ocorrência dessas atividades. Este fato poderia ser revisto, uma vez que, quanto mais frequentemente as ações forem realizadas, em especial as preventivas, mais positivos serão os resultados em relação à prevenção dos agravos à saúde bucal. Portanto, se faz necessária uma maior frequência nas atividades em saúde.

Além disso, as ações educativas foram realizadas, em sua maioria, em escolas e creches. Este dado corrobora com a pesquisa de Venâncio DR et al. (2011) que ressalta que a maior capacidade de aprendizado da criança se dá no período desde o nascimento até o sexto ano de vida. Neste período o cérebro passa por grande parte de seu desenvolvimento. Percebe-se, portanto, que a promoção da saúde em pré-escolares, é um ato relevante em creches e escolas, na medida em que as crianças são capazes de adquirir conhecimento e incorporar hábitos saudáveis precocemente, sendo que tais hábitos podem se prolongar ao longo de sua existência.

\section{CONSIDERAÇÕES FINAIS}

De acordo com os critérios e dados descritos e discutidos no presente trabalho, pode-se concluir que: todos os cirurgiões dentistas entrevistados nas Estratégias de Saúde da Família realizam ações de promoção em Saúde Bucal, com frequência média mensal; as ações de promoção e proteção em saúde bucal das equipes de saúde bucal são realizadas, em sua maioria, pelo cirurgião dentista com auxílio do técnico em saúde bucal. As ações de educação em saúde bucal são desenvolvidas, em sua maioria, utilizando-se recursos audiovisuais como vídeos educativos e álbuns seriados; além da educação em saúde bucal, as escovações supervisionadas são realizadas, predominantemente, em escolas e creches; as ações educativas, campanhas e as escovações são apoiadas integralmente pelos gestores de saúde do município.

\section{AGRADECIMENTOS E FINANCIAMENTO}

Agradecemos a colaboração de todos os entrevistados e a todos que direta ou indiretamente contribuíram para a concretização desse estudo.

\section{REFERÊNCIAS}

1. ALMEIDA GCM, FERREIRA MÂF. Saúde bucal no contexto do Programa Saúde da Família: práticas de prevenção orientadas ao indivíduo e ao coletivo. Cad Saúde Publica, Rio de Janeiro, 2008; 24(9): 2131-2140.

2. AQUILANTE AG et al. The importance of dental health education for preschool children. Rev Odontol. UNESP, Araraquara, 2003; v. 32(1):39-45.

3. BALDINI MH, FADEL CB, POSSAMAI T, QUEIROZ MGS. A Inserção da Saúde Bucal no Programa Saúde da Família no Estado do Paraná. Brasil. Ciência e Saúde Coletiva, Rio de Janeiro, 2003;1(2):27-32

4. BARROS SG, CHAVES SCL. A utilização do Sistema de Informações Ambulatoriais (SIA-SUS) como instrumento para caracterização das ações de saúde bucal Epidemiol. Serv. Saúde, Beasilia,2003; 12(1):41-51

5. BRASIL. Ministério da Saúde. Portaria № 1444/GM/28/12/2000 Seção 1, p. 85 . Incentivo financeiro para a reorganização da atenção à saúde bucal prestada nos municípios por meio do Programa Saúde da Família. Brasília: Ministério da Saúde, 2004.

6. BUISCHI YP. Promoção de saúde bucal na clínica odontológica. São Paulo, Artes Médicas, 2003.

7. BUSS P. Promoção da saúde e qualidade de vida. Ciência \& Saúde coletiva, 2000; 5(1): 163-177. 
8. CARVALHO IVC, CASTRO JGD. Promoção de saúde bucal:práticas dos cirurgiões-dentistas da estratégia da saúde da família do município de palmas tocantins. Desafios, Palmas, 2018; 5(3) 20-29.

9. DUCAN BB et al. Medicina Ambulatorial: Condutas de Atenção Primaria Baseadas em Evidências., 3ª edição, Porto Alegre: Artemed, 2006.

10. ISMAIL AI, BURT AA., EKLUND SA. Epidemiologic patterns of smoking and periodontal disease in the United States. JADA, San Francisco, 1983; 306:617-621.

11. LEAVELL HR, CLARK EG. Medicina preventiva. São Paulo: McGraw Hill do Brasil, 1976.

12. MURRAY JJ, NAYLOR MN. Fluorides and dental caries, pp. 32-67. In JJ Murray (org.). The prevention of oral disease. (3 ed.). Oxford University Press, Nova York (1996).

13. PEREIRA GDS et al. A PROMOÇÃO DA SAÚDE BUCAL NO CONTEXTO ESCOLAR: UMA REVISÃO INTEGRATIVA. Revista Expressão Católica Saúde, Quixadá, 2017; 2(2):9-16.

14. PIUVEZAM G et al. Ações de promoção em saúde bucal: um estudo com o povo indígena Tremembé, CE. ROBRAC. Revista de Odontologia do Brasil Central, 2005; 14(37):60-64.

15. SÁ LO, VASCONCELOS MMVB. A Importância da educação em saúde bucal nas escolas de Ensino Fundamental - Revisão de literatura. Odontologia Clín-Científic, 2009;8(4):299-303.

16. SIGERIST HE. The University at the crossroads. New York: Henry Schumann Publishers (1946).

17. SILVA AH, ROSSONI E, SANTOS UEL. Título do artigo. Braz j periodontol, 2018; 28(2):7-13.

18. SILVA RCL et al. Quimo-Enfermagem. Políticas Pública de Saúde. Rio de Janeiro: Editora Aguia Dourada, 2006.

19. VENÂNCIO DR et al. Promoção da saúde bucal: desenvolvendo material lúdico para crianças na faixa etária préescolar. J Health Sci Inst. São Paulo, 2011; 29(3):153-156.

20. WINSLOW CA. The unitilled fields of public health. Science, $195151(1306): 23-50$. 